\title{
Computer Technology to Improve Medical Information in Bangkok, Thailand
}

\author{
Waraporn Jirapanthong*
}

\author{
College of Creative Design and Entertainment Technology, Dhurakij Pundit University, Bangkok, Thailand
}

\begin{abstract}
Building hospital applications based on services allow hospitals and other organizations to cooperate and make use of business functions. Hospital information systems that involve extensive information exchange across hospitals and organization boundaries, such as patient profiles, can easily be automated. Service-based applications can be constructed by linking services from various providers using either a standard programming language or a specialized workflow language. This paper reviews the use of computer technology which supports health services in Bangkok, Thailand by developing a survey of health services in hospitals, in which the focus is on the attitudes and competence of medical students and physicians, and the availability of health services equipment; and analyzing and providing guidance via a web service that supports health services. A prototype of a web application is created to simulate situations of the use of computer-based devices for supporting clinical operations, involving 12 medical doctors and 3 patients. Two cases are analysed to demonstrate different situations of the web service. In such situations, stakeholders are requested to query patient information and specify the documents. The experiments have been evaluated by considering straightforward criteria to perform activities with the prototype to determine how accurate the documents are requested and specified, and evaluate how the health service performs efficiently.
\end{abstract}

Keywords: E-Hospital Systems, Hospital Application, Web Services, Medical Health Services.

\section{INTRODUCTION}

Many hospitals in Thailand, even in Bangkok, still lack of sophisticated information-based systems due to some difficulties in practical. In addition to, legacy systems are old software systems that are used by a hospital. Usually, they rely on obsolete technology but are still essential to the business. It may not be cost effective to rewrite or replace those systems and many organizations would like to use them cooperating with new systems. Web service becomes a solution to enhance computer systems.

Using a web service, organizations can make their information accessible. Other software systems can access the information by defining and publishing a web service interface. This allows the data being accessed. Although a lot of applications have been converted to allow connecting though web services, computer systems that support health services are not completely satisfied. Our research is also to analyse and design a web service that supports medical staffs and tools in hospitals in Bangkok.

This research aims to survey the use of computer technology which supports health service in Bangkok by developing a survey of health services in hospitals in Bangkok, in which focus on the attitude and competency of medical students and physicians, and

*Address of correspondence to this author at the College of Creative Design and Entertainment Technology, Dhurakij Pundit University, Bangkok, Thailand; Tel: +66 29547300; Fax: +66 29548651; E-mail: waraporn.jir@dpu.ac.th

JEL: C88, L86, D61. availability of health services equipment; and to analyze and provide guidance a web service supporting on health services.

This study focuses on medical doctors and medical students who work for public hospitals in Bangkok. The study was to explore the factors associated with the use of computer technology that supports the treatment and diagnosis of sickness. We focus on participants who have experiences with the use of computer-based devices and tools. Additionally, this study has developed the prototype of a web application to simulate situations of the use of computer-based devices to support the operation of the clinic. The study involves 12 medical doctors and 3 patients.

As the following, the background of the management of information, information technology, and knowledge management that support treatment activities in hospitals are described. The background of and web service are also presented.

\section{BACKGROUND AND RELATED WORK}

\subsection{Information and Computer Technology for Health Service in Thailand}

Recently, there are requirements on information and computer technology such as telemedicine systems that have been applied as an alternative to provide public health service (Pasotti and Zucca, 2014). According to (Asangansi and Aiyetan, 2005), Thailand's medical systems now focus on new telemedicine. The information and computer 
technology (Smith et al., 2003; Moulin et al. 2003; Coiera, 1995; Asangansi and Aiyetan, 2005; Ozumba, 2002; Bello et al., 2004) has played an important role to leverage the public health in Thailand, in particular, it supports providing medical services. New technologies have been added and enhanced health services in Thailand, especially, people who are far away and inconvenient to access the public health service. Network technology and telemedicine have been a part of medical treatment in some regions within upcountry.

Some experimental projects for telemedicine are developed to provide consultation and communication between doctors, hospitals or provincial public health office. For example, experiments with health centers in nine provinces in the North and Northeast of Thailand are created. The health centers have participated in 45 projects and those have received the support from the Ministry of Public Health, Telecommunications of Thailand, and the company QualCom which provides notebooks for provincial medical and health centers. The project has been operated for two years.

Princess Mother's Medical Volunteer Foundation (Princess Mother's Medical Volunteer Foundation Journal 2017) is an important pioneer to drive basic health services to local areas, particularly, to enable local people who have difficulties to access health services. Telemedicine is also used as an alternative way to serve the public.

Moreover, Chulabhorn Research Institute (Biomedical Research 2016) also drives and supports public health services. They have brought up new technology to improve medical services. The institute has worked with the National Electronics and Computer Technology Center (NECTEC) in agreement technical cooperation for research and development. The first phase of telemedicine systems focus on the preparation of the telemedicine system. For example, they try to build up a broadband network to transmit images from the PET-CT to medical professionals. The system facilitates medical staffs to read the results and diagnostic images of cancer cells through computer networks. This is expected to make higher standard of medical technology and more efficient healthcare system in Thailand.

The telemedicine systems have been continuously and steadily developed. One of those is called Medical Grid allows telemedical treatment more powerful. It takes advantage and build a high-speed processing power without high cost of purchasing a computer server. It makes Virtual organization which allows searching information faster and richer.

\subsection{Web Services}

Web services are distributed application components that are externally available. It can be used to integrate computer applications that are written in different programming languages and run on different platforms. Web services are language and platform independent because vendors have agreed on common web service standards. A lot of related standards are developed and proposed by different vendors.

For example, Oracle has developed a java.net project called Metro (Oracle America, Inc. 2013). Metro is a complete web service stack, covering all of a developer's needs from simple "Hello, World" demonstrations to reliable, secured, and transacted web services. Metro includes Web Services Interoperability Technologies (WSIT) (Oracle, Inc. 2010). WSIT supports enterprise features such as security, reliability, and message optimization. WSIT ensures that Metro services with these features are interoperable with Microsoft NET services (Tabor, 2001). Within Metro, Project Tango develops and evolves the codebase for WSIT.

Several programming models are available to web service developers. These models fall into two categories, both supported by the IDE:

i) REST-based (REpresentational State Transfer) (Richards, 2006) is a new way to create and communicate with web services. In REST, resources have URIs and are manipulated though HTTP header operations.

ii) SOAP/WSDL-based (Richards, 2006). In traditional web service models, web service interface are exposed through WSDL documents (a type of XML), which have URLs. Subsequent message exchange is in SOAP. Another type of XML document.

REST-based ("RESTful") web services are collections of web resources identified by URIs. Every document and every process is modeled as a web resource with a unique URI. These web resources are manipulated by the actions that can be specified in an HTTP header. Neither SOAP, nor WSDL are used. Instead, message exchange can be conducted in any format e.g. XML (Richards, 2006), JSON (ECMA 
International 2013). HTML, etc. In many cases a web browser can serve as the client.

HTTP is the protocol in REST. Only four methods are available: GET, PUT, POST, and DELETE. Requests can be bookmarked and responses can be cached. A network administrator can easily follow what is going on with a RESTful service just by looking at the HTTP headers.

REST is a suitable technology for applications that do not require security beyond what is available in the HTTP infrastructure and where HTTP is the appropriate protocol. REST services can still deliver sophisticated functionality. For example, Flickr (Yahoo! Inc. 2013), Google Maps and Amazon all provide RESTful web services.

In SOAP-based web services, Java utilities create a WSDL file based on the Java code in the web services. The WSDL is exposed on the net. Parties interested in using the web service create a Java client based on the WSDL. Messages are exchanged in SOAP format. The range of operations that can be passed in SOAP is much broader than what is available in REST, especially in security.

SOAP-based web services are suitable for heavyweight applications using complicated operations and for applications requiring sophisticated security, reliability or other supporting features. They are suitable when a transport protocol other than HTTP has to be used. Many of Amazon's web series, particularly those involving commercial transactions, and the web services used by banks and government agencies are SOAP-based.

The Java API for XML Web Services (JAX-WS) is the current model for SOAP-based web services in Metro. JAX-WS is built on the earlier HAX-RPC model but uses specific Java EE features such as annotations to simplify the task of developing web services. Because it uses SOAP for messaging, JAX-WS is transport neutral.

As mentioned earlier, a traditional web service is based on SOAP. A web service is an instance of a more general notion of a service, which is defined (Lovelock et al., 1996) as: "an act or performance offered by one party to another. Although the process may be tied to a physical product, the performance is essentially intangible and does not normally result in ownership of any of the factors of production".
As shown in Figure 1, the web service consists of

i) SOAP (Simple Object Access Protocol). This is a message interchange standard which facilitates the communication between web services. The protocol is provided for communicating among diverse platforms or programming languages. This is supported by XML technology.

ii) WSDL (Web Service Definition Language). This is a standard for service interface definition. It defines how to use web services and describes types of sending and receiving messages.

iii) UDDI (Universal Description, Discovery and Integration). This defines the components of a service specification, which may be used to discover the existence of a service.

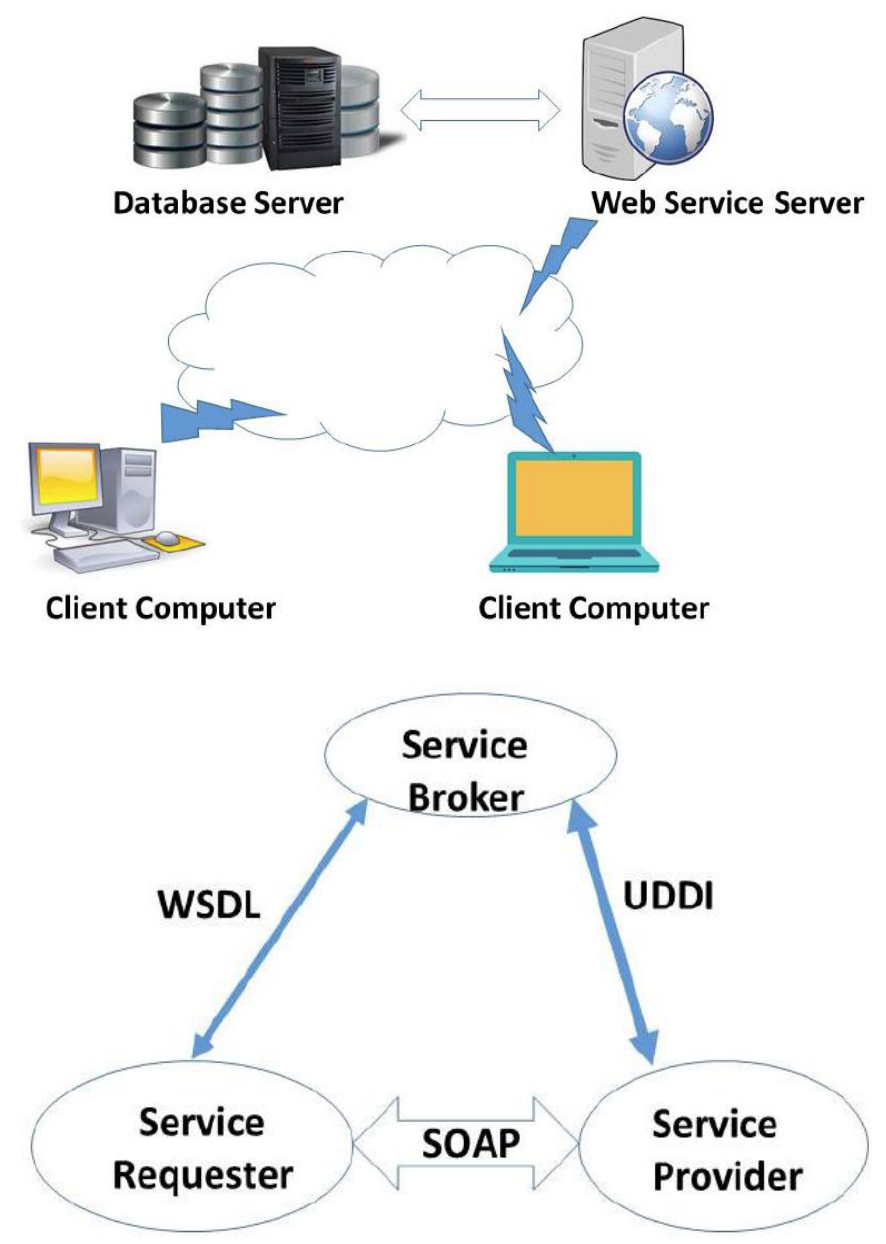

Figure 1: Service-oriented architecture.

The web service is available at a web service server, which internally connects a database server and externally connected to the Internet. The web service can be accessed by client computers via the Internet. 


\section{RESEARCH METHODOLOGY}

\subsection{Population and Sample}

In this study, the target population was a group of medical doctors. We planned to develop a survey of the use of computer technology which supports the health services in Bangkok. The survey has taken in public hospitals in Bangkok and participated by medical doctors from those hospitals. This study focuses on medical doctors who work for public hospitals in Bangkok. There were 12 medical doctors and 3 patients participating.

\subsection{How to Collect Data}

We have done practical survey on 3 public hospitals in Bangkok. It consists of three parts.

i) For the first part, we performed an observation and used a questionnaire to collect primitive data. From the observation, it captures the details of working environment that was taken four places (departments) in two hospitals. From questionnaire, the primitive data includes personal and working profiles of respondents, the attitude and behavior towards working environments, and the use of computer systems at work, especially the use of Internet applications regarding medical perspectives. The distribution of questionnaire was randomly performed and there were 23 respondents. The questionnaire consisted of open and closed questions.

ii) For the second part, a research team has studied for an indicator of disease severity in patients before, during and after symptoms through the brain and heart. The data from the survey is used to develop a tool to distinguish the important indicators in the brain. We have proposed to apply those indicators in the prototype. Particularly, we have developed a web service that supports medical staffs, particularly on first-aid treatment for stroke patience by applying the indicators.

iii) For the third part, we have performed an observation and used a questionnaire to collect data and feedback on the use of the prototype. The prototype is our proposed web service. From the observation, it captures the emotional perspectives on the use of prototype. From questionnaire, the primitive data includes personal and working profiles of respondents, the attitude and feedback on using the prototype. The questionnaire was selectively distributed and there were 12 respondents. The questionnaire consisted of open and closed questions.

\subsection{Methods}

We have conducted the analysis as described below.

\section{a. Develop the Survey on Public Hospitals}

The objectives are to:

i) collect the primitive data includes personal and working profiles of respondents

ii) acquire the attitude and behavior towards working environments, and the use of computer systems at work, especially the use of Internet applications regarding medical perspectives.

iii) identify a set of indicators of disease severity in patients before, during and after symptoms through the brain and heart in order to be applied in the prototype.

\section{b. Create and Define the System Requirements}

The requirements are further used for developing the prototype. As shown in Figure 2, an E-Hospital Web Service is designed to support different providers in different places in which offer facilitating services. A legacy system of hospitals are analysed and the design of e-hospital web service. One of objectives of the service is that it is not necessary to decide when the system is programmed or deployed what service provider should be used or what specific services should be accessed.

As the hospital system is operated, the information system uses the service to find the most appropriate information service and binds to that. Because of the use of Health monitoring system, it can access database systems across hospitals and therefore make local information available to medical staffs who do not personally contact the hospitals.

The first subsystem is monitoring system which serves as an elementary monitoring system that is responsible for reviewing all telemetry orders to determine if the patient meets criteria for hard telemetry or soft telemetry. This is based on established 


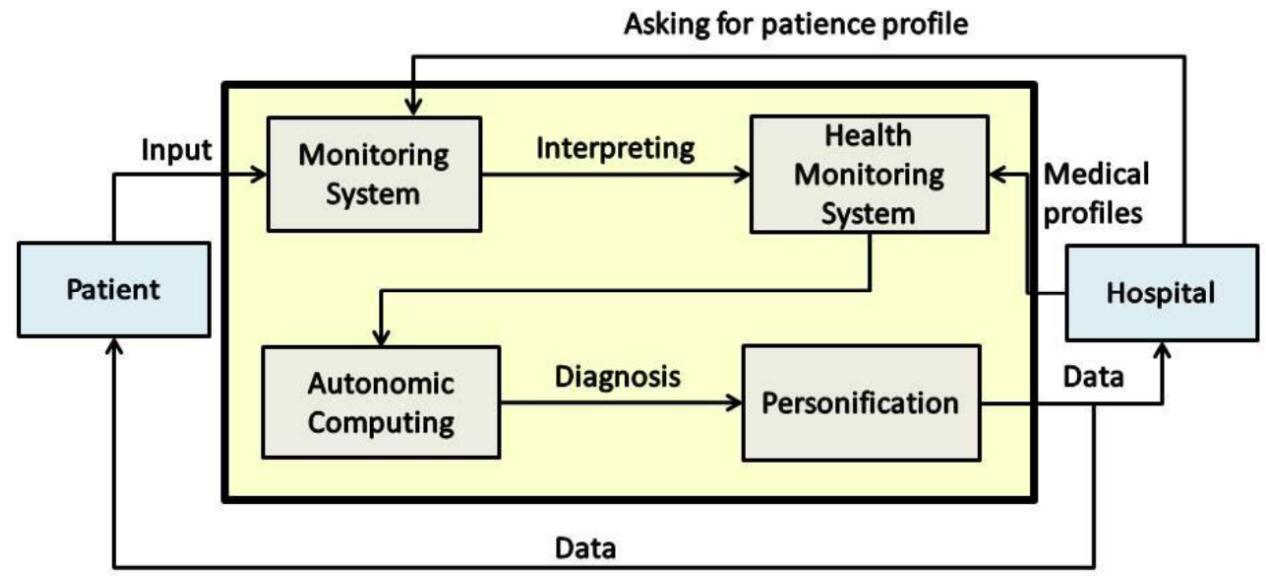

Figure 2: E-Hospital Web Service.

guidelines. And this requires thorough review of a patient's current condition and previous medical history.

For the second subsystem, Health monitoring system will serve medical staff as a consultant for cardiopulmonary technicians who monitor and interpret cardiac physiological data, detecting and documenting any changes in hear rhythm and arrhythmias according to department standards.

The next subsystem is the autonomic computing. This subsystem computes the cardiovascular system that maintain blood supply to vital organs, particularly the brain. This requires information regarding blood pressure, which can be maintained in the short term by altering systemic resistance and cardiac output.

The last subsystem is a part of decision support system to assist the medical staffs to make a decision to maintain or cover the patient's urgent sickness situations.

Based on the survey of hospitals in Bangkok, we found that there are information systems available in the hospitals. However, those systems lack of data continuity. It becomes difficulties to follow patients' profiles and medical records.

Our approach, for the part of database, we have designed two main parts. Firstly, patient profile- the data is aimed to identify patients. Secondly, patient behavior- the data is aimed to monitor and automatically diagnose the patient disease. The steps of e-hospital web service can be described as follows:

i) authentication - this step is to identify and grant the access right for a user. This step is performed via web server service. ii) collect data from database server. This step is performed by database server through web service broker.

iii) SOAP data encryption.

\section{c. Developing a Prototype}

In our approach, we have developed a web service which is applied with web service protocols including SOAs and service information exchange (SOAP). We have also applied programming language based on XML. The templates of elementary artifacts are created i.e. XSD and XSLT.

WSDL Specifications can be automatically generated. Service Requests do not need to know the details of a specification. An example of a WSDL specification which provides service show in Figure 3. It shows a part of defining some templates used. We assume that the namespace prefix "xs" refers to the namespaces URI for XML schemas and the namespace prefix "hp" associated with the following definition regarding the hospital domain. As shown in Figure 3, it shows the part of the description of the element and type definition that is used in the service specification. This defines the elements firstname, lastname, and gender.

According to the system requirements, we have designed the templates of XML Schema, WSDL description and other XML-based documents. In our experiments, we have created XML-based documents regarding our approach.

\section{EXPERIMENTS AND EVALUATION}

\subsection{Overview}

We have performed an observation and used a questionnaire to collect data and feedback on the use 


$$
\begin{aligned}
& <\text { patients }> \\
& <\text { xs: } \text { schema targetNameSpace }=\underline{\text { http: } / / . . \text { ehospital } x m l n s: h p}= \\
& \text { http://../ehospital> } \\
& <\text { xs:element name }=\text { "BirthDate" type }=\text { "patientrec" } /> \\
& <\text { xs:element name }=\text { "CitizenID" type }=\text { "patientrec" }> \\
& <\text { xs:element name }=\text { "HN" type }=\text { "orgrec" } /> \\
& <\text { xs:element name }=\text { "SocialInsuranceID" type = "pubrec" } /> \\
& \text {... } \\
& <\text { xs:complexType name }=\text { "patientrec" }> \\
& <\mathrm{xs} \text { :sequence }> \\
& <\text { xs:element name }=\text { "firstname" type }=\text { "xs:string" }> \\
& <\text { xs:element name }=\text { "lastname" type }=\text { "xs:string" } \mid> \\
& <\text { xs:element name }=\text { "gender" type }=\text { xs:string" }> \\
& \text {... } \\
& </ \text { xs:sequence }> \\
& </ \text { xs:complexType }> \\
& \text {... } \\
& <\text { xs:schema }> \\
& </ \text { patients }>
\end{aligned}
$$

Figure 3: Part of a WSDL description for a web service.

of the prototype, the web service. From the observation, it captures the emotional perspectives on the use of prototype. The questionnaire was selectively distributed and there were 12 respondents. The questionnaire consisted of open and closed questions. Those questions are based on the perspective of the use of a prototype to support their present job.

Moreover, some medical doctors were interviewed to determine the effectiveness of the existing facilities maintenance management practices and processes. Thirty two questionnaires were distributed to medical staffs in the hospitals. Twelve out of thirty two questionnaires were completed. The distributions of the respondents were from three public hospitals and involved three patients.

The questionnaire comprised of seven parameters of equipment life cycle which was adequately answered. The questionnaire had two main parts; Section $A$ and Section B. In section A, the questionnaire defines the goal of the research and outlines the seven main parameters to be interviewed on. In section $B$, each main parameter had several questions in which the facility maintenance managers were required to answer appropriately. The weighting of each question was answered based on the following tabulation;

$\begin{array}{llll}0 & \text { - } & \text { Very poor: } & 0 \% \\ 1 & - & \text { Poor: } & 20 \% \\ 2 & - & \text { Fair: } & 40 \% \\ 3 & - & \text { Good: } & 60 \% \\ 4 & \text { - } & \text { Very good: } & 80 \% \\ 5 & - & \text { Excellent: } & 100 \%\end{array}$

Additionally, we have developed a prototype tool to facilitate the demonstration and evaluation of the approach. The prototype tool has been implemented by applying with Java-based and XML-based technologies. The main functionalities of the tool are implemented according to the design as shown in Figure 2. Additionally, two cases are created to demonstrate different situations of the web service, 
involving (a) different types of documents; and (b) different patients and medical staffs. The experiments of document creation have been evaluated by considering three criteria: (i) easy, (ii) correctness, and (iii) completeness. For the latter criteria, the precise and recall measurements are used.

\subsection{Results and Analysis}

According to the questionnaire, the questions are used to collect feedback on the use of the prototype. From 20 questions of questionnaire with different weights per each question, 12 respondents gave slightly similar feedback. The average weighted score of 20 questions from 12 respondents are $60.2727 \%$. The figure shows that the satisfaction of the use on the prototype is good.

Additionally, we have simulated the situations of the use of computer technology to support the medical treatment and consultation. Particularly, we have established the case study for this purpose. The objectives of two cases are varied. The main objective of test case 1 is to identify the patient profiles across hospitals via the web service. The data used are collected from 2005-2006 and there are 3,751 records available. The results of test case 1 show that correctly identified is $87.93 \%$ while misidentified is $47.6 \%$. The percentage of correctly identified is high (87.93\%). It implies the web service performs the identification of patient profiles with high performance.

The percentage of misidentifying is fairly low $(47.6 \%)$. It implies the service failed to identify some profiles. This is due to incomplete data for example, missing some attribute values, incorrect data. Additionally, the main objective of test case 2 is to use the patient profiles to diagnose the patients' diseases. The data used are collected from 2004-2006 and there are 4,652 records available. The result of test case 2 show that correctly diagnosis is $83.2 \%$ while misdiagnosis is $55.2 \%$.

A number of possible directions for further investigations have been identified. We provide in this section future work of the research, what needs to be done to improve the approach and to increase the benefits of the approach. Tools for Document Generation and Visualisation for large number of various artefacts should be implemented. It is therefore believed that the approach could benefit by providing tool fully support for the specification of documents. In addition, sophisticated techniques for visualization could support the use of documents more efficiently.

\section{CONCLUSION AND FUTURE WORK}

According to modern technologies and the environments are being changed rapidly. Also many environment factors cause health problems and lead to being sick from disease and symptoms. Based on our survey, many hospitals in Thailand, even in Bangkok, still lack of sophisticated information-based systems due to some difficulties in practical.

In addition to, legacy systems are old software systems that are used by a hospital. It may not be cost effective to rewrite or replace those systems and many organizations would like to use them cooperating with new systems. Web service becomes a solution to enhance computer systems. This allows the data being accessed. Although a lot of applications have been converted to allow connecting though web services, computer systems that support health services are not completely satisfied.

This research have done the survey regarding the use of computer technology which supports health service in Bangkok and proposed a guidance a web service supporting on health services.

This study focused on medical doctors and medical students who work for public hospitals in Bangkok And it explored the factors associated with the use of computer technology that supports the treatment and diagnosis of sickness. This study has developed the prototype of a web application to simulate situations of the use of computer-based devices to support the operation of the clinic. Particularly, the study has created a case study of symptoms, particularly, an indicator of disease severity in patients before, during and after symptoms. Also, the prototype has been to support medical staffs, particularly on first-aid treatment for stroke patients by applying the indicators.

The experiments showed the results in one direction. To conclude, we found that the computer technology in hospitals can support health services in hospitals in Thailand. We believe that the application of such technology to operation of the clinic is to provide effective support. The results of this study showed that it is capable to continue the development of computer technology to perform the clinical efficiency further.

Additionally, a number of possible directions for further investigations have been identified. The future work can be considered in order to improve the approach and to increase the benefits of the approach. Those are: 
i) The approach is planned to be expanded to cover all activities in hospitals in order to complete the web service for e-hospital framework.

ii) It is believed that the approach could benefit by providing tool support for the specification of documents.

iii) We plan to improve the algorithm of implementation to reduce of processing time. Since, transactions on web service can take a long time to be processed depending on the size, number, and types of documents and relationships, more work needs to be done to optimize the processing time.

\section{ACKNOWLEDGEMENT}

The author wishes to thank Chia-Lin Chang and Michael McAleer for helpful comments and suggestions.

\section{REFERENCES}

Asangansi, I.E., Aiyetan, P.O. 2005. "Public Health and Bioinformatics." Dokita 30(1):24-27.

Bello, I.S., et al. 2004. "A Knowledge and Utilization of Information Technology Among Health Care Professionals and Students in Ile-Ife, Nigeria: A Case Study of a University Teaching Hospital." Journal of Medical Internet Research 6(4):e45. https://doi.org/10.2196/jmir.6.4.e45

Biomedical Research. 2016. Retrieved July 4, 2016, from Chulabhorn Research Institute, Web site: https://www.cri.or.th/en/
Coiera, E. 1995. "Medical informatics." BMJ Journal 310:1381-1386. https://doi.org/10.1136/bmj.310.6991.1381

ECMA International. 2013. "JSON: The Fat-Free Alternative to XML." Retrieved November 13, 2016, Web site: http://www.json.org/xml.html

Lovelock, C. et al. 1996. "Developing Global Strategies for Service Businesses." California Management Review 38(2):64.

Moulin, T., Retel, O., Chavot, D. 2003. "Impact of new information and communication technologies (NTIC) on hospital administration and patient management." Care Network for Diagnosing and Treating Neurologic Emergencies April:191200.

Oracle America, Inc. 2013. "Metro." Retrieved July 4, 2016, Web site: httpfs://metro.java.net/

Oracle, Inc. 2010. "Web Services Interoperability Technology (WSIT)." Retrieved September 17, 2016, Web site: https://docs.oracle.com/cd/E19681-01/8203740/ghdcp/index.html

Ozumba, B.C. 2002. The World Congress on the Internet in Medicine. MEDNET. Available at: http://www.mednet2002.org/ abstracts/display.cfm?id=63180901

Pasotti, L., Zucca, S. 2014. "Advances and Computational Tools towards Predictable Design in Biological Engineering." Computational and Mathematical Methods in Medicine 11:116. https://doi.org/10.1155/2014/369681

Princess Mother's Medical Volunteer Foundation Journal. 2017 Retrieved January 17, 2017, from Princess Mother's Medical Volunteer Foundation, Web site: http://www.pmmv.or.th/ index.php/th/

Richards, R. 2006. Pro PHP XML and Web Services. APress.

Smith J.J., Mallard-Smith, R.J., Beattie, V., Beattie, D.K. 2003. "Use of information technology in general practice." Journal of the Royal Society of Medicine 96(8):395-397. https://doi.org/10.1258/jrsm.96.8.395

Tabor, R. 2001. Microsoft .NET XML Web Services. Sams.

Yahoo! Inc. 2013. "Flickr." Retrieved November 13, 2016, Web site: https://www.flickr.com/

Received on 16-02-2017

\section{DOI: https://doi.org/10.6000/1929-7092.2017.06.28}

(C) 2017 Waraporn Jirapanthong; Licensee Lifescience Global.

This is an open access article licensed under the terms of the Creative Commons Attribution Non-Commercial License (http://creativecommons.org/licenses/by-nc/3.0/) which permits unrestricted, non-commercial use, distribution and reproduction in any medium, provided the work is properly cited. 